\title{
CEF Regimen
}

National Cancer Institute

\section{Source}

National Cancer Institute. CEFRegimen. NCI Thesaurus. Code C138100.

A chemotherapy regimen consisting of oral cyclophosphamide, epirubicin and fluorouracil, used as a treatment for recurrent and metastatic breast cancer. 